\title{
Ultraminiature optical design for multispectral fluorescence imaging endoscopes
}

Tyler H. Tate

Molly Keenan

John Black

Urs Utzinger

Jennifer K. Barton 


\title{
Ultraminiature optical design for multispectral fluorescence imaging endoscopes
}

\author{
Tyler H. Tate, ${ }^{a, \star}$ Molly Keenan, ${ }^{b}$ John Black, ${ }^{c}$ Urs Utzinger, ${ }^{a, b}$ and Jennifer K. Barton ${ }^{a, b}$ \\ aUniversity of Arizona, College of Optical Sciences, Tucson, Arizona, United States \\ bUniversity of Arizona, Biomedical Engineering, Tucson, Arizona, United States \\ ${ }^{\circ}$ Glannaventa Inc., San Mateo, California, United States
}

\begin{abstract}
A miniature wide-field multispectral endoscopic imaging system was developed enabling reflectance and fluorescence imaging over a broad wavelength range. At 0.8-mm diameter, the endoscope can be utilized for natural orifice imaging in small lumens such as the fallopian tubes. Five lasers from 250 to $642 \mathrm{~nm}$ are coupled into a $125-\mu \mathrm{m}$ diameter multimode fiber and transmitted to the endoscope distal tip for illumination. Ultraviolet and blue wavelengths excite endogenous fluorophores, which can provide differential fluorescence emission images for health and disease. Visible wavelengths provide reflectance images that can be combined for pseudo-white-light imaging and navigation. Imaging is performed by a $300-\mu \mathrm{m}$ diameter three-element lens system connected to a 3000-element fiber. The lens system was designed for a 70-deg full field of view, working distance from $3 \mathrm{~mm}$ to infinity, and $40 \%$ contrast at the Nyquist cutoff of the fiber bundle. Measured performance characteristics are near design goals. The endoscope was utilized to obtain example monochromatic, pseudowhite-light, and composite fluorescence images of phantoms and porcine reproductive tract. This work shows the feasibility of packaging a highly capable multispectral fluorescence imaging system into a miniature endoscopic system that may have applications in early detection of cancer. ๑ 2017 Society of Photo-Optical Instrumentation Engineers (SPIE) [DOI: 10.1117/1.JBO.22.3.036013]
\end{abstract}

Keywords: multispectral imaging; fluorescence; lens design; endoscope; cancer; obstetrics/gynecology

Paper 160727PR received Oct. 21, 2016; accepted for publication Mar. 6, 2017; published online Mar. 23, 2017.

\section{Introduction}

Early detection and diagnosis of some diseases is limited by the ability to obtain high-resolution images in deeply buried organs. An example is ovarian cancer, the deadliest female reproductive malignancy. ${ }^{1}$ Ovarian cancer typically presents with nonspecific symptoms and current screening methods (physical examination, transvaginal ultrasound, the CA-125 blood test) have proven not to be reliable or effective when used for annual screening in the general population in large clinical trials. ${ }^{2-4}$ Detection at a stage early enough to make a significant difference in outcome is problematic both because of the lack of obvious symptoms and the peculiar natural history of the most lethal phenotype of the disease-high-grade serous ovarian cancer (HGSOC). ${ }^{5,6}$ The model suggests that HGSOC exists in an occult asymptomatic form for 3 to 4 years, where it is too small to be detected by a secreted biomarker, magnetic resonance imaging, computed tomography, and probably ultrasound, followed by a rapid growth phase in years 4 to 5 with concomitant presentation of symptoms, at which time the tumor is already metastatic and lethal. ${ }^{7,8}$

Emerging consensus suggests that a significant fraction, perhaps over $50 \%$, of HGSOC actually originates in the fallopian tube with subsequent migration to the ovary and peritoneum. ${ }^{9,10}$ If this hypothesis is correct it opens a window on early detection of the disease through minimally invasive endoscopy using relatively inexpensive but robust optical components. Optical imaging enables localization of disease and could confirm

*Address all correspondence to: Tyler H. Tate, E-mail: TylerTate@email. arizona.edu diagnosis from a suspicious blood marker before invasive surgical procedures are required. High-resolution optical techniques potentially have the capability to yield both structural and functional information at the resolution level necessary for early detection but free-space optics cannot penetrate trans-dermally through the body to sufficient depth. Fiber optics and miniaturization of free-space components allow integration into minimally invasive endoscopes.

The body provides a natural pathway via the vagina, cervix, and uterus to access the fallopian tubes and ovaries. The proximal tubal opening of the fallopian tube is located in the uterus with an ostium diameter of $\sim 1 \mathrm{~mm} .{ }^{11}$ The tube then expands to a maximum intratubal diameter of $\sim 1 \mathrm{~cm}$ throughout a 12- to $15-\mathrm{cm}$ tortuous path to the fimbriae where the tube is attached to the ovary. ${ }^{11}$ Simple white-light imaging endoscopes called falloposcopes with outer diameters (ODs) less than a millimeter have been used previously to image the fallopian tube in vivo for fertility-impeding blockages. ${ }^{12-15}$ These falloposcopes were introduced through the working channel of a hysteroscope. Imaging was performed by a lens attached to a coherent fiber bundle (CFB). Falloposcopes designed for tubal imaging of fertility blockages lack sufficient imaging performance to screen for subtle changes presented by early-stage ovarian cancer.

The navigational portion of a screening falloposcope should be forward-viewing with a wide-field of view (FOV) and in a flexible package of at most $800-\mu \mathrm{m}$ OD. Forward-viewing, wide-field endoscopes have three primary technologies to relay images from the distal tip to the computer: fiber bundles, miniature detectors, and optical scanning. Many modern endoscopes

$1083-3668 / 2017 / \$ 25.00$ @ 2017 SPIE 
now use miniature CMOS detectors directly in the endoscope tip. Miniature detectors with $0.5 \mathrm{~mm} \times 0.5 \mathrm{~mm}$ dimensions have been demonstrated and $1 \mathrm{~mm} \times 1 \mathrm{~mm}$ detectors are commercially available. ${ }^{16}$ As the technology continues to shrink, it may be viable for an $800-\mu \mathrm{m}$ OD endoscope, but is not currently an option. Scanning techniques such as piezo scanning fiber (SF) endoscopes have also continued to shrink. ${ }^{17}$ A $1.2-\mathrm{mm}$ implementation has been used to image $1 \mathrm{~cm}$ of the distal fallopian tube in vivo. ${ }^{18}$ The technique provides significant optical advantages and high-effective pixel density, but requires additional miniaturization to be inserted farther into the fallopian tube. The most traditional forward-viewing endoscopic imaging technique uses a CFB. A lens in the endoscope tip images onto the distal face of the CFB and a separate imaging system outside the body then images the proximal face of the CFB onto a detector. For endoscopes with submillimeter diameters, the CFB approach remains the most viable technology but as with any camera system there is a trade-off between FOV and resolution. The limited size of the falloposcope restricts the number of CFB pixels compromising the ability to perform simultaneous wide-field navigation with near cellular resolution. However, fluorescence imaging can enable detection of early disease through tissue functional changes.

Fluorescence imaging can be used to visualize changes in chemical make-up of tissue and is well suited to wide-field imaging. In particular endogenous, fluorophores (tryptophan, collagen, nicotinamide adenine dinucleotide + hydrogen, etc.) can have different concentrations in normal and abnormal tissue. Using UV and short-wave visible excitation sources, different fluorophores can be excited in the tissue. Endogenous fluorescence differences among normal, benign, and cancerous ovarian tissues have been characterized both in vivo and ex vivo by investigators and others using fiber-optic probes ${ }^{19-22}$ and widefield autofluorescence imaging. ${ }^{23-25}$ Mutispectral fluorescence imaging (MFI) can image in both reflectance and fluorescence mode to either measure optical absorption or fluorescence emission, both of which can be an indicator of molecular changes (changes in concentration of hemoglobin or fluorophores) or to combine multiple reflectance images for pseudo-white-light imaging.

Dual-modality optical coherence tomography (OCT) and fluorescence imaging endoscopes have been demonstrated previously. A 2.1-mm diameter SF endoscope for multispectral fluorescence imaging of exogenous fluorophores has been demonstrated $^{26,27}$ without UV excitation or OCT capabilities. A preliminary study had limited success performing in vivo white light imaging of the fallopian tube with a 1.2-mm SF endoscope. ${ }^{18}$ The larger diameter device had difficulty passing through the tube. Side-viewing needle probes with diameters of a few hundred microns have also been demonstrated with OCT only ${ }^{28}$ and using a dual-clad fiber for OCT and single-point fluorescence detection. ${ }^{29}$ Other endoscopes of a few millimeters in diameter have combined various implementations of fluorescence imaging with OCT. ${ }^{30-33}$ None of these examples combines widefield fluorescence imaging and OCT into a submillimeter diameter package. Furthermore, none of these examples includes UV excitation to use MFI for wide-field autofluorescence detection.

Miniaturizing wide-field MFI for endoscopic use is the next step to validate use of the technique in vivo. In this paper, we describe the optical design and characterization of a miniaturized MFI system and its integration into an 800- $\mu \mathrm{m}$ diameter falloposcope prototype.

\section{Design and Methods}

\subsection{Optical Design}

The MFI optical design is comprised of four optical subsystems with independent and interrelated specifications (Fig. 1). Following the path of the light the four optical subsystems are: proximal illumination, distal illumination, distal imaging, and proximal imaging. For effective navigation, the overall system specification for full field of view (FFOV) is $70 \mathrm{deg}$. Based on previous studies, the included illumination wavelengths are 250, 375, 406, 515, and $642 \mathrm{~nm} .{ }^{19-25}$ Laser sources are used for the endoscope prototype and are similar in central wavelength to those provided by a filtered xenon arc lamp in our previous MFI imaging study on ex vivo ovarian and fallopian tube tissue. ${ }^{25}$ The ultraviolet and blue wavelengths efficiently excite the endogenous fluorophores. Sampling the blood absorption curve in reflectance mode with 375,406 , and $515 \mathrm{~nm}$ can also provide diagnostic utility. ${ }^{25}$ Finally, pseudo-white-light imaging can be obtained by acquiring and combining a sequence of images with the 406-, 515-, and 642-nm illumination wavelengths. The broad spectral range including $250-\mathrm{nm}$ light and the requirement to perform both reflectance and fluorescence imaging requires separate excitation and illumination channels. The imaging channel is optimized for imaging from 375 to $642 \mathrm{~nm}$. Thus, 250 -nm illumination is used only for fluorescence mode, but all other illumination wavelengths can be used in either reflectance or fluorescence mode. In order to accommodate integration into a flexible endoscope with OD of

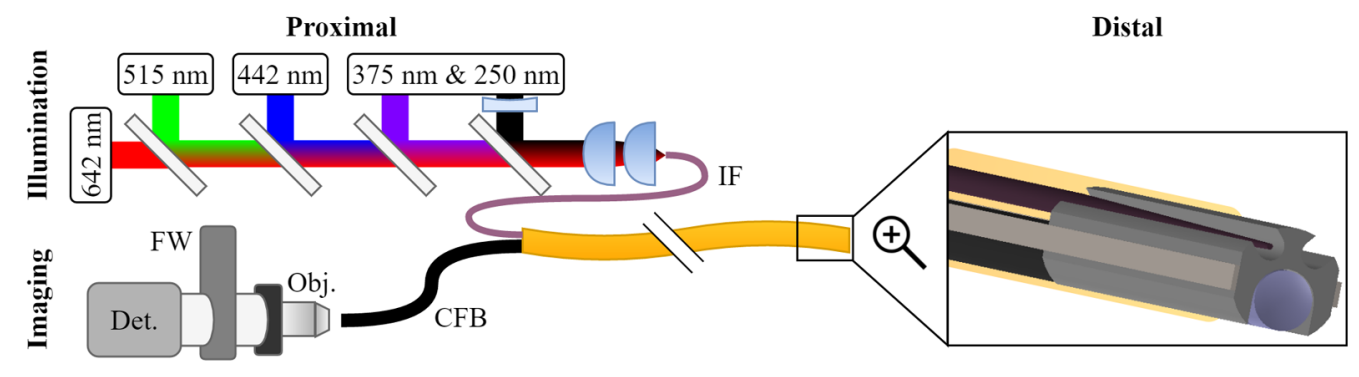

Fig. 1 Endoscopic MFI system overview. The proximal illumination system combining five-laser beams into the illumination fiber (IF) is shown top left. The proximal illumination system imaging the proximal face of the CFB through the microscope objective (Obj.), filter wheel (FW), and onto the detector (Det.) is shown bottom left. On the right is a zoomed in model of the distal tip showing the tapered IF on top and the imaging lens with CFB on bottom, both flush with the distal end of the ferrule. A protective cover plate is not shown. 
at most $800 \mu \mathrm{m}$, the illumination and imaging channels are limited to 125 - and $300-\mu \mathrm{m}$ diameters, respectively. The remaining space is necessary for the ferrule, pull-wires, and protective sheath. Mechanical and steering details of an endoscope with similar mechanical design, but without MFI capabilities, have been published previously. ${ }^{34}$ However, the design does allow a second $125-\mu \mathrm{m}$ diameter lumen for additional future functionality without exceeding total OD requirements.

\subsubsection{Proximal illumination}

Proximally, the five-laser beams are collimated, co-aligned, and focused into the illumination fiber (IF). The two ultraviolet wavelengths are produced by a pulsed, frequency doubled, and tripled alexandrite laser described previously. ${ }^{35}$ The 406-, 515-, and 642$\mathrm{nm}$ wavelengths are provided by single mode pigtailed diode lasers (Models: LP406-SF20, LP520-SF15, and LP642-SF20, Thorlabs, Newton, New Jersey) each with a temperaturecontrolled diode driver (CLD1010LP, Thorlabs). Each of the visible lasers is collimated by an adjustable focus collimator (CFC11X-A, Thorlabs). The beams are combined using a series of four dichroic mirrors (Models: ZT594RDC, ZT488RDC, ZT375RDC, Chroma Technology Corp, Bellows Falls, Vermont, and model FF310-DiO1, Semrock Inc., Rochester, New York). The 642-nm laser is the reference beam and passes through all dichroics. Each subsequent shorter wavelength is coaligned with the red beam using a dichroic that transmits the longer wavelength(s) and reflects the shorter wavelength such that after the fourth dichroic all five-laser beams are coaligned.

The coaligned beam path is then focused into the IF. Based on the diameter and high UV to Vis transmission requirements, the IF used was a high-OH silica core fiber (FIP100110125, Polymicro, Phoenix, Arizona) with 0.22 numerical aperture (NA), a $100-\mu \mathrm{m}$ core and an OD of $125 \mu \mathrm{m}$. No autofluorescence from the IF was detected that would reduce the system signal to noise ratio. The primary coupling lenses are a pair of uncoated plano-convex 20-mm focal length calcium fluoride lenses (LA5315, Thorlabs). Using a pair of lenses rather than a single lens provides two major advantages at the cost of one disadvantage. The split lenses significantly reduce spherical aberration, necessary to focus the lasers into the $100-\mu \mathrm{m}$ core fiber. Additionally, the 4- to 4.5-mm diameter laser beams require an effective focal length of $\sim 10 \mathrm{~mm}$ to match the 0.22-NA fiber. The effective focal length of the lens pair is $9.8 \mathrm{~mm}$ and the $20-\mathrm{mm}$ focal length lenses are readily available off-the-shelf, whereas an equivalent single lens is not. However due to the wide spectral range, the lenses are uncoated and using a pair of lenses increases losses from Fresnel reflections. Despite calcium fluoride being low dispersion, the lenses require longitudinal chromatic aberration correction. The focus location of the 375-nm beam is used as the reference focal distance. The adjustable focus collimators on the visible beams are effectively used as weak positive lenses to independently match each visible beam focus to the $375-\mathrm{nm}$ beam focus. The $250-\mathrm{nm}$ beam is chromatically corrected by placing a negative $200-\mathrm{mm}$ UV antireflection-coated fused silica lens (48-325, Edmund Optics, Barrington, New Jersey) in the beam path just prior to dichroic mirror. Assuming 4.5-mm beam diameters the design theoretically focuses all five beams to the same location with root mean square radii between 5.5 and $15.5 \mu \mathrm{m}$ and matching the IF. The $100-\mu \mathrm{m}$ core, 22-NA fiber is placed just beyond focus to couple in the light without damaging the fiber face. This simple design provides adequate performance and reasonable transmission using inexpensive spherical optics for coupling laser light into the IF. Excellent transmission would be possible using a single-coated asphere.

\subsubsection{Distal illumination}

The distal illumination must fully illuminate the FFOV imaged by the distal imaging objective. Multimode fibers with the necessary spectral transmission range and OD are not available with sufficient NA to illuminate a 70-deg FFOV. Thus, the IF distal output illumination angle requires modification. The IF is not coaxial with the imaging optics axis, so the illumination angle must be slightly larger than the 70-deg imaging field. Thus, the desired output NA is at least 0.6 , corresponding to a 73.7-deg illuminated FFOV. The étendue of a fiber is approximately constant meaning the output NA can be increased as the area of output face of the fiber is decreased. Reducing the fiber face area to $36.7-\mu \mathrm{m}$ diameter increases output NA to 0.6. The distal end of the fiber is tapered to meet this specification. ${ }^{34}$

The prototype design does not currently include a cover plate on either the illumination or imaging fibers. Preliminary analysis suggests that using a single-cover plate over the illumination and imaging channels will cause back reflections from the IF off the cover plate to enter the imaging channel for illumination NA greater than $0.25 .{ }^{36}$ For a clinical device, the optical channels will need protection. This could be done either by using separate cover plates for the illumination and imaging channels or by using a single-cover plate with an extruded channel for the IF.

\subsubsection{Distal imaging}

To provide good navigational performance through the fallopian tube and on the surface of the ovary, the distal imaging lens design must image a 70-deg FFOV and have a long depth of focus. Additionally, the lenses must image a wide spectral range of 375 to $642 \mathrm{~nm}$ to acquire fluorescence and reflectance images. Physically, the lens design is limited to $300-\mu \mathrm{m}$ diameter elements with reasonable thicknesses and curvatures to reduce manufacturing and construction complexity.

The resulting optical design is a custom three-element lens (Fig. 2) that images onto a 3000-element imaging CFB (Fujikura FIGH-03-215S). From distal to proximal, the lens element materials are S-FPM3, S-FPL51Y, and Sapphire. For initial proof-ofconcept validation, the lenses are uncoated. The CFB has a

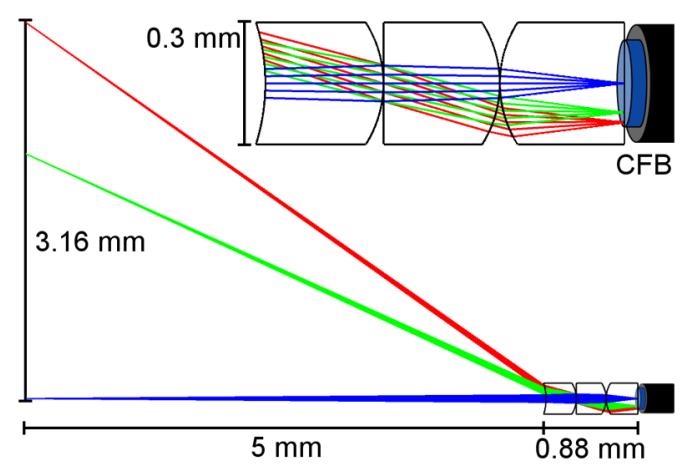

Fig. 2 Custom three-element distal imaging lens. The zoomed out view (bottom) shows the 6.38-mm FFOV at a nominal working distance (WD) of $5 \mathrm{~mm}$. The total lens length is $0.88 \mathrm{~mm}$ and the lens $O D$ is $0.3 \mathrm{~mm}$. A magnified view of the lenses focusing onto the CFB is also shown (top). The image circle of the CFB (blue) has a diameter of $0.19 \mathrm{~mm}$. 
coating diameter of $285 \mu \mathrm{m}$, image circle diameter of $190 \mu \mathrm{m}$, $0.4 \mathrm{NA}$ and typical core-to-core spacing of 3.1 to $3.4 \mu \mathrm{m}$. The attenuation of light increases significantly for wavelengths shorter than $390 \mathrm{~nm}$, but over a length of at most a few meters necessary for an endoscope is usable at $375 \mathrm{~nm}$. The number of elements in the CFB fundamentally limits the system spatial resolution. A 3000-element CFB has provided sufficient image resolution in the fallopian tube in past fertility endoscopes. ${ }^{12-15}$

The minimum 3.1- $\mu \mathrm{m}$ core spacing of the CFB corresponds to a maximum Nyquist cutoff frequency of 161 cycles $/ \mathrm{mm}$. The resulting lens design is $\mathrm{f} / 2.4$ and achieves $40 \%$ modulation transfer function (MTF) contrast at the Nyquist cutoff of the CFB for polychromatic imaging for all rays over a 70-deg FFOV and at all working distances from $3 \mathrm{~mm}$ to infinity. While outside optimal range, contrast remains above $30 \%$ for working distances as short as $1.2 \mathrm{~mm}$. An MTF contrast above $20 \%$ at the frequency cutoff of the fiber should be sufficient for imaging. The lens has been designed with an extra performance buffer to account for alignment tolerances discussed below. The designed polychromatic MTF at a nominal working distance of $5 \mathrm{~mm}$ is shown in Fig. 3. In most circumstances, the lens will be used to image single wavelength reflectance or specific fluorescence emission bands rather than the full design wavelength range. The MTF is further improved in these scenarios as much of the degradation comes from chromatic aberrations over the full spectral range. The system can be calibrated at each wavelength so that images acquired sequentially at different wavelength bands can be digitally color corrected when fused for analysis.

Normal mounting and alignment techniques are nearly impossible to implement at the miniature scale requiring sufficient imaging performance to be maintained with moderate construction alignment tolerances. The elements were assumed to have a maximum radius and thickness deviation of $30 \mu \mathrm{m}, 0.2$ fringes of spherical and astigmatism irregularity, index error of 0.001 and Abbe deviation of $1 \%$. Construction tolerances were

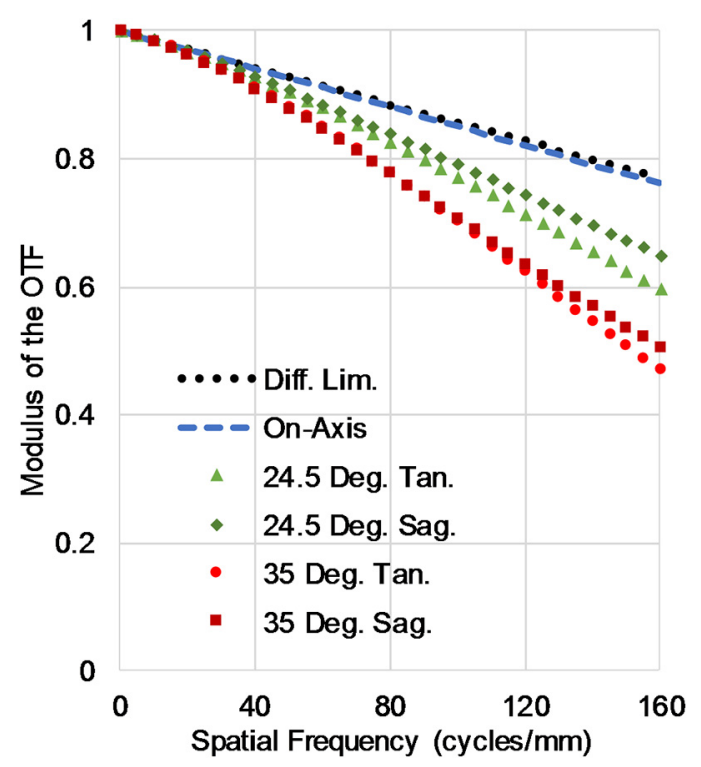

Fig. 3 Polychromatic MTF of the three-element lens design at a working distance of $5 \mathrm{~mm}$. The vertical axis is the modulus of the optical transfer function and the horizontal axis is spatial frequency in cycles per millimeter. The minimum core-to-core spacing of the fiber bundle corresponds to a maximum frequency cutoff of $\sim 160$ cycles per millimeter. determined to be a maximum $30 \mu \mathrm{m}$ of decenter and $1 \mathrm{deg}$ of tilt in either axis for all elements. A 1000 run Monte Carlo sensitivity analysis was performed using these parameters at the nominal working distance of $5 \mathrm{~mm}$ and wavelength of $450 \mathrm{~nm}$. The performance criterion measured was the average MTF at the frequency cutoff at 161 cycles $/ \mathrm{mm}$, which has a nominal value of 0.629 . Without any focus compensation, the mean MTF after tolerancing is expected to be 0.597 with $90 \%$ of trials exceeding 0.554 . In other words, even with moderate construction tolerances, the average contrast at the frequency cutoff is expected to drop $<8 \%$ in over $90 \%$ of builds and $<3 \%$ in more than $50 \%$ of builds. Thus, the lens system should maintain adequate imaging performance out to the cutoff frequency of the fiber after construction.

The design prioritizes correction of spherical aberration, coma, axial color, and astigmatism. Distortion is the most significant remaining aberration followed by field curvature and lateral color. These aberrations are acceptable as resolution (and thus detection ability) is more important than faithful reproduction of a flat, even field while imaging the nonplanar geometry inside the fallopian tube. Each individual image uses a single excitation wavelength and narrow emission band. Thus, lateral color will not be an issue within each image, but rather something that can be corrected with software for composite image combination. Distortion does not reduce image sharpness and is an acceptable trade-off for the wide 70-deg FFOV. Another design feature includes being image-space telecentric, to provide even illumination onto each fiber in the $\mathrm{CFB}$ and maintain a constant image circle diameter of $190 \mu \mathrm{m}$ to match the CFB.

The three elements in the lens are stacking and include only two unique nonplanar surfaces. The stacking design simplifies construction by allowing the lenses to be dropped into the MFI imaging channel of the ferrule and held proximally by the fiber bundle and distally by a cover plate. Using only two unique nonplanar curvatures reduces costs by simplifying construction and testing for the manufacture. The length of the lens assembly is $0.88 \mathrm{~mm}$ allowing ample space to secure the CFB behind the lenses inside a 3-mm-long ferrule. This rigid length is not expected to compromise tracking and navigation in tortuous anatomy.

\subsubsection{Proximal imaging}

The proximal face of the fiber bundle is imaged with a modified microscope. An infinity-corrected, UV-enhanced, $20 \times$ objective (UApo/340 $20 \times / 0.75$, Olympus, Tokyo, Japan) is used with a UV-corrected achromat tube lens of focal length $151.5 \mathrm{~mm}$ (027-3025, OptoSigma, Santa Ana, California). Olympus objective magnifications are specified assuming a 180-mm focal length tube lens. The shorter focal length tube lens results in a reduction in overall magnification from $20 \times$ to $16.8 \times$. The detector (PIXIS 1024, Princeton Instruments, Acton, New Jersey) has $13 \times 13 \mu \mathrm{m}$ pixels with typical single pixel well depth of $100 \mathrm{ke}^{-}$. In this configuration, the $3.1-\mu \mathrm{m}$ core-tocore spacing of the CFB is $52.2 \mu \mathrm{m}$ on the detector corresponding to $\sim$ four detector pixels per core-to-core spacing, exceeding the Nyquist sampling requirement. In the infinity space between the objective and tube lens, a 10-position filter wheel (FW) (LB10W-3985, Sutter Instrument, Novato, California) allows specific emission bands for fluorescence imaging to be selected, or an open position allows for reflectance imaging. The minimum switching time between filter positions is $55 \mathrm{~ms}$. 


\subsection{Image Processing}

Images were recorded as 16-bit grayscale images using WinView (Princeton Instruments). Individual images were processed using image analysis software (ImageJ, National Institutes of Health) and MATLAB (The MathWorks, Natick, Massachusetts). For all images, the pixel values outside the fiber bundle image circle were set to 0 . The pixels inside the image circle were contrast enhanced such that the brightest $1 \%$ and darkest $1 \%$ of pixels were, respectively, set to the maximum and minimum intensity values. After contrast enhancement, a slight Gaussian blur was applied to increase visual perception by mitigating the pixilation effects of the CFB without degrading resolution. ${ }^{37}$ Color reflectance images were created by weighted combinations of 406-nm illumination for the blue channel, 515-nm illumination for the green channel, and 642-nm illumination for the red channel. Weighting in each channel was qualitatively adjusted to match perception of accurate color rendition. Color fluorescence images used the light collected from narrow band 450-, 532-, and 590-nm emission filters. All emission filters had approximate fullwidth half-maximum of $10 \mathrm{~nm}$. Composite images were created by combining the three emission filter images and qualitatively weighting the channels to approximate visual color intensity.

\subsection{Imaging System Testing}

The MFI imaging system resolution and field-of-view were characterized and compared to theoretical simulations. Additionally, the imaging system's ability to create a pseudowhite light image and to capture fluorescence images was evaluated.

\subsubsection{Imaging resolution testing}

The imaging lens parameters and approximate fiber bundle parameters were modeled as a complete system (Imager, Five Focal, Boulder, Colorado) to determine the expected minimal resolvable feature size and FOV at working distances of 1, 3, 5,7 , and $10 \mathrm{~mm}$.

The constructed endoscope imaged bar targets on USAF test target at the center and edge of the field for each working distance. The test target was trans-illuminated with white light and images were acquired in grayscale without an emission filter. The resulting images were processed as described above with a Gaussian blur radius of 1.4 pixels.

The resolution was evaluated on-axis and at the edge of the field by imaging a series of bar targets near the resolution cutoff at each location. The edge of the field was considered approximately nine tenths of the radius to reasonably fit the bar targets into the FOV. A representative profile was drawn across horizontal and vertical bars of each target and the modulation frequency was measured. The Rayleigh resolution criteria for two points specify a drop of $26.4 \%$ between peak and valley intensity. Modulation contrast is given by the following equation:

modulation contrast $=\frac{\left(I_{\max }-I_{\min }\right)}{I_{\max }+I_{\min }}$.

Thus, the Rayleigh criteria correspond to a modulation contrast of $15.21 \%$. Bar targets with modulation contrast for both vertical and horizontal bars greater than or equal to $15.21 \%$ were considered resolvable. The width of the minimum resolvable bar was compared to the modeled minimum resolvable feature size for each working distance.

Testing with white-light illumination should provide results similar to the polychromatic MTF in Fig. 3. In practice, the system is designed to primarily image narrowband fluorescence or reflectance. The frequency cutoff of the CFB fundamentally limits the maximum resolution at any given working distance. However, narrowband imaging will reduce the chromatic aberrations and thus increase the MTF at the frequency cutoff of the CFB, particularly at high frequencies off-axis. Therefore, the resolution is not expected to improve in narrowband imaging configurations, but contrast and thus image quality may improve.

\subsubsection{Imaging field of view testing}

The constructed endoscope's FOV was measured by imaging a square-wave target with one line-pair per millimeter at each working distance. The target was trans-illuminated with white light and images were acquired in grayscale without an emission filter. The line pairs were used to measure the approximate FOV in millimeters at each working distance and the compared to the simulated values.

\subsubsection{Fluorescence standards imaging}

The endoscope's ability to image fluorescence was evaluated by imaging cuvettes of sulforhodamine, fluorescein, and quinine sulfate (Table 1). The stable and well-characterized dyes are ideal for testing. They fluoresce over a similar wavelength range as biological soft tissue and are diluted to fluorescence with intensity similar to tissue autofluorescence. The three cuvettes were placed side-by-side and the five-color illumination laser beam was aimed to pass through all three vials. The imaging endoscope was placed perpendicular to the laser beam such that it could image the laser beam path inside each vial. For each of the illumination wavelengths except $642 \mathrm{~nm}$, three images were recorded with narrow band emission filters at 450, 532, and $590 \mathrm{~nm}$. A fourth image was recorded without an emission filter. The images were processed as described above. A qualitative composite color image was created using the filtered images. For the composite color image, the 590-nm filter image was mapped to the orange color given by hexadecimal feb900 to match visual perception of the fluorescence signal. The 450- and 532-nm images were mapped to the blue and green channels, respectively.

Table 1 The fluorescence dyes used for testing and their concentration and dissolution solvent.

\begin{tabular}{lcc} 
Dye & Solvent & Concentration $(\mu \mathrm{M})$ \\
\hline $\mathrm{SR}$ & Ethanol & 0.8 \\
QS & $0.5 \mathrm{M} \mathrm{H}_{2} \mathrm{SO}_{4}$ & 2 \\
$\mathrm{FI}$ & $0.1 \mathrm{M} \mathrm{NaOH}$ & 0.2 \\
\hline
\end{tabular}

Note: SR, sulforhodamine; QS, quinine sulphate; and FI, fluorescein. 


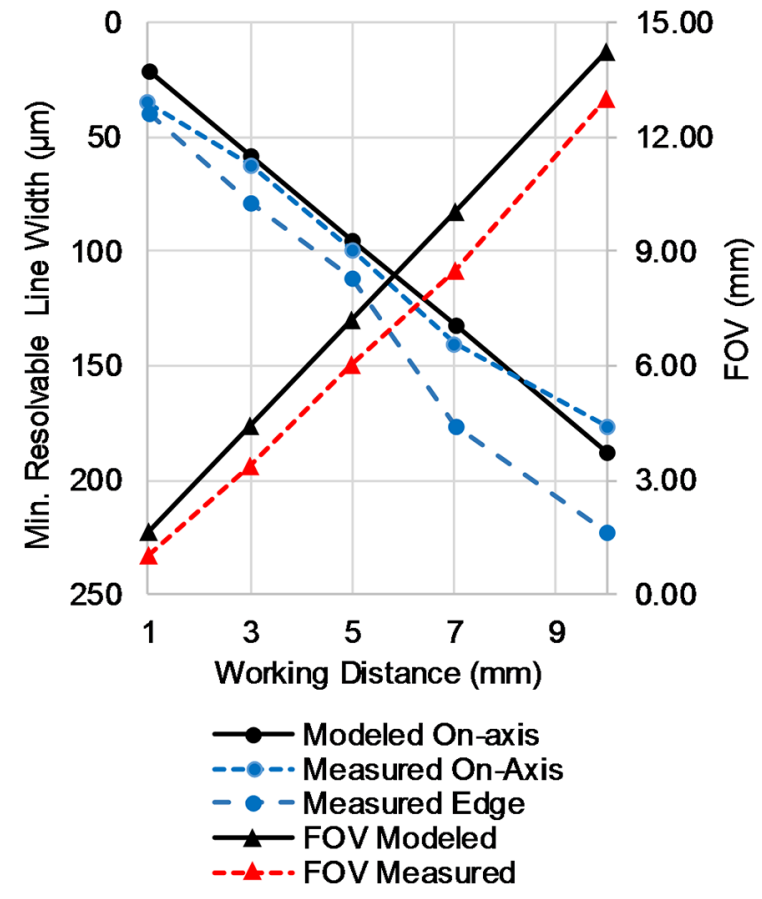

Fig. 4 Measured resolution and FOV compared to modeled values at working distances of $1,3,5,7$, and $10 \mathrm{~mm}$.

\section{Results}

\subsection{Imaging Resolution and Field of View}

Between the optimal working distance range of 3 to $10 \mathrm{~mm}$, the measured minimum resolvable feature size compared favorably with the modeled values (Fig. 4). Sample images of on-axis bar target testing at 3, 5, and $10 \mathrm{~mm}$ are shown in Fig. 5 . At a working distance of $1 \mathrm{~mm}$, the endoscope barely resolved the smallest bars (group 3, element 6) on the test target on-axis. Off-axis performance at all working distances was worse than on-axis as expected from the MTF. The FOV was measured at the same working distances as the resolution. It was consistently slightly smaller than the modeled values as shown in Fig. 4.

\subsection{Fluorescence Imaging}

The results of imaging fluorescein, quinine sulfate, and sulforhodamine can be seen in Fig. 6 and the absorption characteristics of the dyes are given in Table 2. Due to the significant
Table 2 Absorption and emission properties for each fluorescence dye. Absorption percentages for each dye are given for each illumination wavelength. Percentages are normalized for each dye such that the peak absorption wavelength is $100 \%$. Emission spectrum peak wavelengths and spectral width at half maximum are also provided.

\begin{tabular}{llcc}
\hline & \multicolumn{3}{c}{ Dye } \\
\cline { 2 - 4 } & SR & QS & FI \\
\hline \multirow{4}{*}{$250 \mathrm{~nm}$} & \multicolumn{2}{c}{ Absorption (\%) } \\
$375 \mathrm{~nm}$ & 14.5 & 100.0 & 29.1 \\
$406 \mathrm{~nm}$ & 5.3 & 15.1 & 1.6 \\
$515 \mathrm{~nm}$ & 1.5 & 0.4 & 1.8 \\
\hline & 9.5 & 0.0 & 13.1 \\
Peak & \multicolumn{2}{c}{ Emission (nm) } & \\
FWHM & 593 & 449 & 38 \\
\hline
\end{tabular}

Note: SR, sulforhodamine; QS, quinine sulfate; FI, fluorescein.

variables that account for final image intensity including illumination power and bandwidth, illumination light absorption, fluorophore quantum yield and concentrations, fluorescence emission spectra, emission filter spectra, optical system spectral response, and image processing, this figure is not quantitative. Qualitative comparison of the fluorescence intensity of the dyes at each illumination wavelength aligns well with predictions based on the approximate quantum yields. The emission filters also successfully blocked the out-of-band illumination light that can be seen in the images with visible illumination and no emission filter. The UV excitation images without a bandpass filter have an IR filter in the active FW location to suppress a small residual amount of the 755-nm alexandrite fundamental beam present in the UV beams.

\subsection{Color Imaging}

In order to test the RGB imaging modality, colorful objects were imaged sequentially with the visible illumination sources and the reflectance images were combined into a color composite

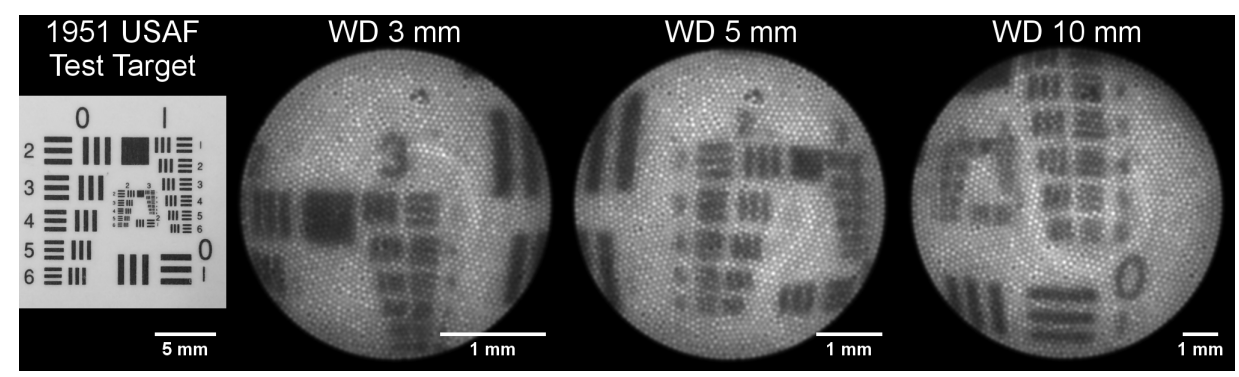

Fig. 5 Sample images from on-axis resolution testing. The left most image is a digital camera image of the 1951 USAF test target used. The following three images from left to right are images of the test target acquired using the endoscope at working distances of 3,5 , and $10 \mathrm{~mm}$. Minimum resolvable features were determined to be group 3 element 1 , group 2 element 3 , and group 1 element 5 , respectively. 


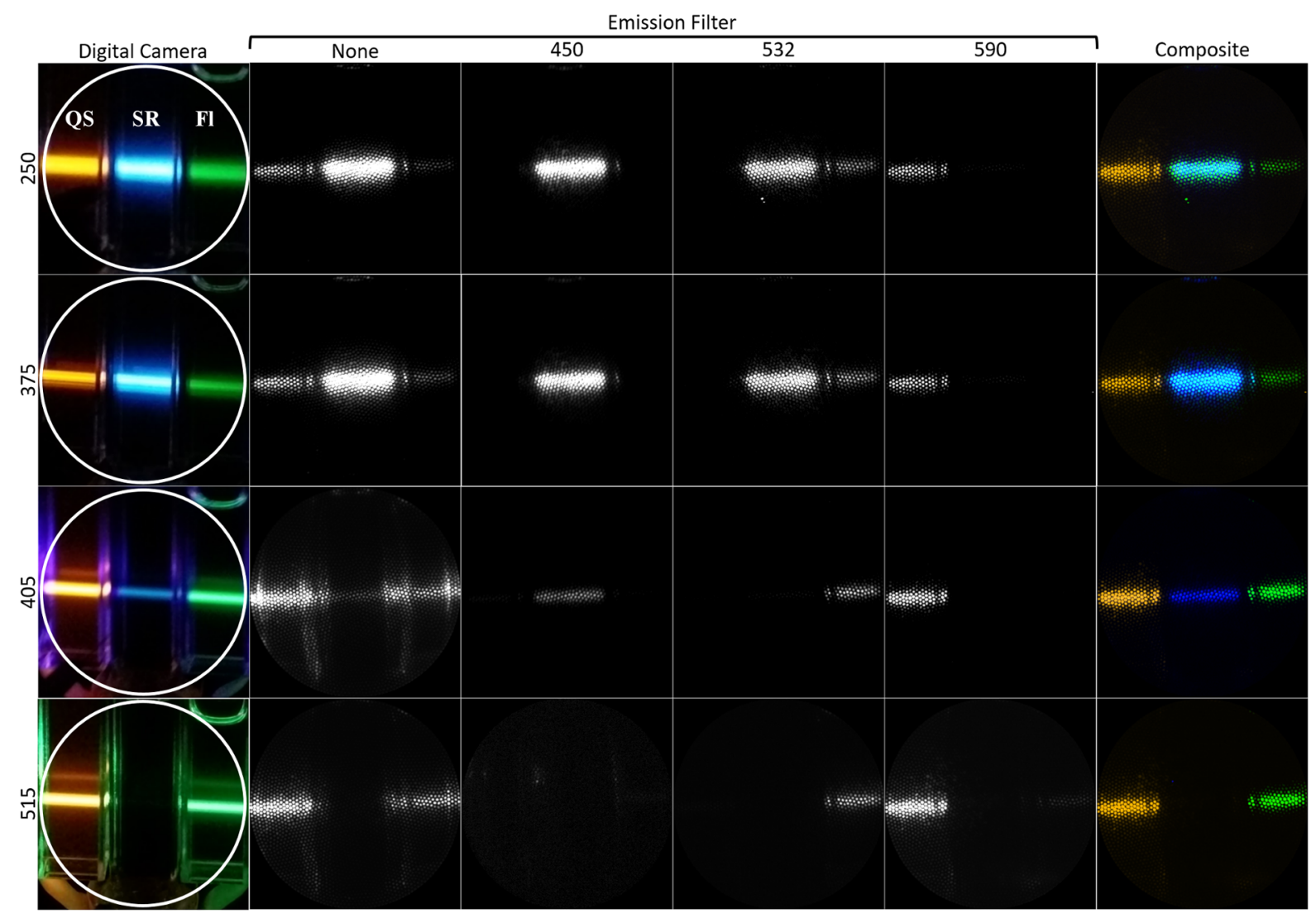

Fig. 6 Images of fluorescence dyes in 1-cm wide cuvettes. From left to right the cuvettes contain sulforhodamine, quinine sulfate, and fluorescein. Each row of images corresponds to one of the four illumination wavelengths used. The left most column is an image taken with a digital camera. The white circle represents the approximate FOV of the endoscope. From left to right, the middle four columns are images taken with the endoscope with no, 450-, 532-, and 590-nm emission filters. The right-most column is the color composite image of the 450-, 532-, and 590-nm emission filter images.

using ImageJ. Figure 7 shows a color composite image of the University of Arizona logo on a business card showing faithful reproduction of the saturated red and blue. Color fluorescence imaging was also tested by imaging a section of porcine ovarian and fallopian tube tissue (Fig. 7). The tissue was acquired from local slaughterhouses in a manner approved by the University of Arizona's Institutional Animal Care and Use Committee. The tissue was illuminated with $375-\mathrm{nm}$ light and imaged sequentially with the 450-, 532-, and 642-nm emission filters. The three fluorescence images were then combined into a composite color image.

\section{Discussion}

A miniature endoscope imaging system capable of wide-field navigation using UV and visible illumination for either autofluorescence or pseudo-white-light imaging modalities has been built. The fully constructed endoscope integrates $300-\mu \mathrm{m}$ diameter lenses and independent illumination channel into a 790- $\mu \mathrm{m}$ OD package for use in the human fallopian tube. The wide FOV with good imaging performance over a large depth of field is ideal for navigation. The ability to perform autofluorescence imaging may allow for increased sensitivity to cancer compared to a standard white-light camera. Achieving the imaging specifications within the specified form factor and over the desired wavelength range posed significant challenges, however, the demonstrated prototype met specifications and has a modular, flexible design for future endoscopes to build upon.

The small and flexible endoscope could be modified for use in other applications such as insertion through a small bore hypodermic needle. The mechanical design of the endoscope allows for steering capabilities as well as an additional $125-\mu \mathrm{m}$ lumen for additional functionality such as high-resolution OCT imaging. The illumination system can be customized to include any laser wavelengths from $250 \mathrm{~nm}$ through the visible spectrum as needed for the application. Near-infrared illumination could also be incorporated into the system, but if used with $250-\mathrm{nm}$ illumination may require custom dichroic mirror coatings. Off-the-shelf dichroic mirrors designed to reflect $250 \mathrm{~nm}$ and transmit longer wavelengths typically have high transmission losses in the near-infrared. A system performing exclusively fluorescence imaging may be able to combine illumination and imaging into a single path for an even smaller form factor. However, UV illumination wavelengths would be limited by $\mathrm{CFB}$ attenuation in a single-path configuration.

The imaging lens and ferrule materials cannot exhibit fluorescence when exposed the UV illumination. Tissue autofluorescence signals are typically very low and any additional fluorescence from materials would reduce signal-to-noise ratio to unacceptable levels. This requirement currently eliminates plastics or polymers used in injection molding, 3-D printing, diamond turning, or GRIN materials. The UV wavelength 


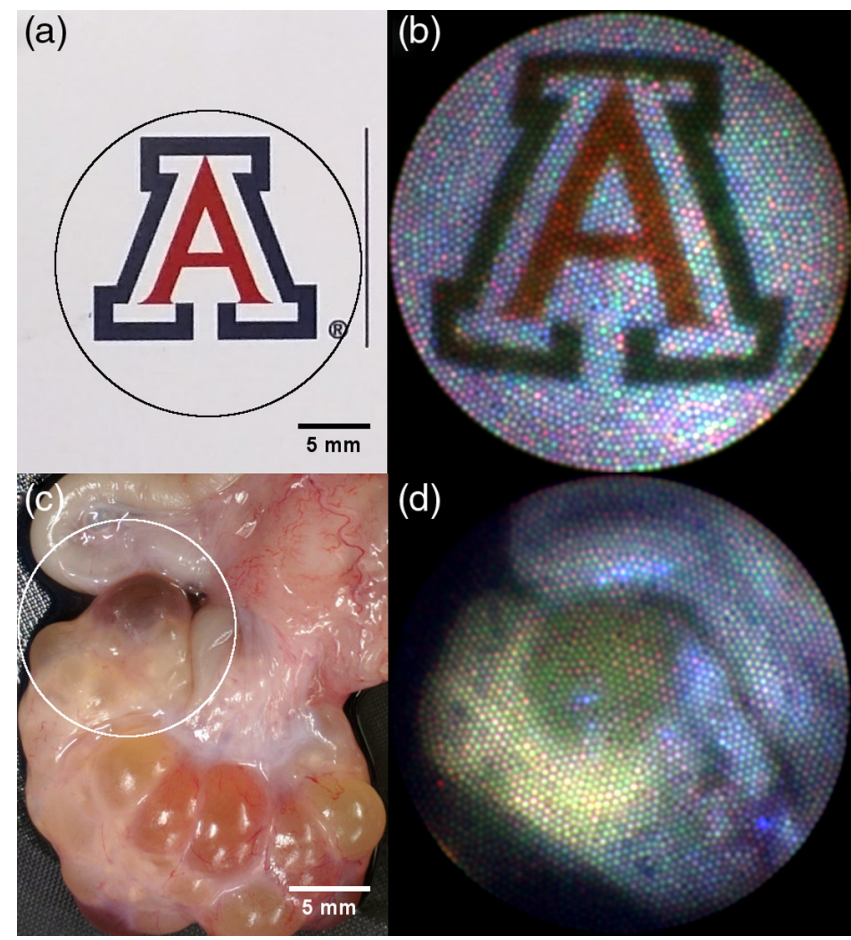

Fig. 7 Images on left are taken using a digital camera and are of (a) the University of Arizona logo on a business card and (c) a section of a porcine reproductive system including ovary with follicles (bottom) and fallopian tube (top left). Corresponding images taken with the endoscope are on the right with FOV approximated by circles overlaid on left images. Image $B$ is a composite color reflectance images taken by sequentially acquiring images with 405-, 515-, and 642-nm illumination. Image D is a composite color fluorescence image created using 375-nm excitation and sequentially acquiring images using the 450-, 532,- and 590-nm emission filters.

requirement also limits the proximal imaging system. Assuming a CFB with reasonable UV transmission becomes available, the system that relays the face of the CFB to the detector would require a specialized microscope objective and tube lens to transmit well below $340 \mathrm{~nm}$. Chromatic aberrations and detector spectral response range could be additional issues when imaging over a wider spectral range. Limited choices of UV transmissive fibers necessitated the need to taper the distal tip of the IF to achieve sufficient illumination angle.

While the endoscope achieved the modeled resolution, the FOV was smaller than expected. The discrepancy could be due to a number of reasons. The most likely cause is the image circle size of the CFB. It is specified to be $190+20 \mu \mathrm{m}$. The lens design is image space telecentric. This feature minimizes angle-of-incidence dependence of the CFB and provides even illumination across the image. The telecentricity also means the 70-deg field is designed to image onto a $190-\mu \mathrm{m}$ diameter circle. Thus at a 5-mm working distance, the expected FFOV is $7.22 \mathrm{~mm}(66 \mathrm{deg})$. If the image circle is reduced to $170-\mu \mathrm{m}$ diameter, then the imaged FFOV would be $5.72 \mathrm{~mm}$ (58 deg) whereas a $210-\mu \mathrm{m}$ image circle would correspond to a FFOV of $7.91 \mathrm{~mm}(75 \mathrm{deg})$. The measured FFOV at 5 -mm working distance is $6 \mathrm{~mm}$, which is within the tolerance range. Changes in fiber bundle image circle diameter will have a trade-off with resolution. The number of fibers remains constant so a smaller image circle will have a decreased average core-to-core spacing of fibers and thus increased resolution potential. This is consistent with the favorable measured against modeled resolution and could explain the better than modeled resolution performance at the $10-\mathrm{mm}$ working distance. The theoretical performance of the lens is overdesigned so a well-aligned lens system would still be fiber-limited out to an image circle size of $220 \mu \mathrm{m}$, which corresponds to an 8.65-mm FFOV at 5 -mm WD $(80 \mathrm{deg})$. Larger fields would begin to be clipped by the edge of the proximal-most lens, which could cause undesirable stray light reflections. Other factors that could contribute to the reduced FFOV are lens alignment and lens manufacturing tolerances.

The ultimate goal of the endoscope is to be used as an effective screening method for ovarian cancer detection and other diseases in tissue that can be reached via small diameter lumens (e.g., pancreatic or bile duct). Achieving that goal is a multistep process. The first generation endoscope presented here successfully proved that MFI imaging can be miniaturized to provide wide-field functional imaging in a sub-800- $\mu$ m diameter package. The next step is to improve the design such that robust endoscopes can be built quickly and reliably to provide safe real-time imaging in limited human trials. Optical engineering challenges for the second-generation system are to simplify, shrink, and automate the proximal optical systems.

For the use in the surgical environment, the endoscope needs to be robust and sterilizable while the proximal system needs to be compact and portable for integration into a surgical cart. Simple, compact UV sources, particularly for wavelengths shorter than $315 \mathrm{~nm}$ have previously been lacking. The UV wavelengths in the current prototype are provided by a large and in-house customized laser. ${ }^{35}$ While UV-B and UV-C sources with sufficient beam quality to deliver in a compact fiber remain challenging to implement, recent developments in diode and DPSS lasers as well as LED technology have made compact, robust, and low-power UV-A sources a reality. It is now possible to build a very compact source with wavelengths from near-UV to the NIR. Some newer sources allow sufficiently short on/off modulation so that the process of capturing a sequence of images synced to detector acquisition for each illumination wavelength can be easily automated by a computer.

The proximal imaging optics currently make use of a modified microscope stand with a 10-position high-speed FW. The design allows rapid swapping of emission filters to optimize wavelength combinations using the prototype device at the expense of real-time imaging. The ideal image combinations presented during operation need further development with input of gynecological surgeons. Once the ideal filter bands are determined, using the prototype system, a second-generation proximal imaging system optimized for real-time imaging, can be built.

For example, the image in Fig. 7(d) (porcine false color fluorescence) required three images each with 60-s exposure to acquire using narrowband ( $10 \mathrm{~nm}$ FWHM) emission filters. A previous ex vivo MFI tissue imaging study in the lab successfully used emission filters with bandwidths of 35 to $100 \mathrm{~nm}$. Replacing the interference filters with commercially available high-transmission $50+\mathrm{nm}$ bandwidth filters will increase the light collection efficiency of the proximal system an order of magnitude. Another order-of-magnitude gain in imaging speed can be realized by replacing the FW and monochromatic detector with a snapshot multispectral imaging camera capable of imaging all wavelength bands simultaneously. ${ }^{38}$ Purchasing or building such a system was outside the scope of this prototype 
build. The advantage in imaging speed is actually twofold. A straightforward decrease in image acquisition time occurs from collection of all spectral information in a single exposure and elimination of the time delay from filter switching. The maximum safe exposure limit to the excitation light must account for only a single exposure rather than multiple. Thus, the second advantage in imaging speed of this technique is safely increasing the illumination intensity to further decrease the necessary exposure time for sufficient light collection. A final advantage of a simultaneous acquisition is the elimination of motion artifacts between sequential images. Further gains in light collection efficiency can be made by adding AR coatings to the distal and proximal MFI optics. Adding a 1\% loss coating to each surface in the distal lens and proximal tube lens (air spaced doublet) would decrease Fresnel reflection losses from $\sim 41 \%$ to $10 \%$. These three primary changes as well as other small optimizations will be necessary to achieve real-time MFI imaging in a second-generation device.

Several government regulatory bodies and other organizations, including the International Commission on Non-Ionizing Radiation Protection (ICNIRP), publish radiation exposure limits for the eyes and skin. Pending further studies on internal tissues these are the best available guidelines. The limits for 375 - and 250-nm light are 39,000 and $7 \mathrm{~mJ} / \mathrm{cm}^{2}$, respectively. ${ }^{39}$ The power density will be highest for tissue closer to the endoscope tip. Assuming tissue is exposed to UV radiation at a working distance of $1 \mathrm{~mm}$ for a maximum of $1 \mathrm{~min}$ during a screening procedure, the limits on average laser power are $5.1 \mathrm{~mW}$ at $375 \mathrm{~nm}$ and $0.001 \mathrm{~mW}$ at $250 \mathrm{~nm}$. The porcine tissue in Fig. 7(d) was imaged with 375-nm excitation with a similar average laser power, but at a working distance of $\sim 10 \mathrm{~mm}$. At the longer working distance, the estimated total exposure to acquire the three sequential images is $<2 \%$ of the limit at $375 \mathrm{~nm}$ and could be further reduced by implementing the above optimizations. Meeting exposure limits at 250-nm excitation would be challenging and will require optimization of system efficiency.

The device presented here achieved initial proof-of-concept objectives. Implementing the discussed changes in a second generation will allow limited human trials to further validate and refine the technology. Long-term, a large clinical trial on high-risk patients with a third-generation device will be necessary to determine real-world efficacy as a regular screening technique.

\section{Disclosures}

The authors report grants from the United States Department of Defense, the National Institute of Biomedical Imaging and Bioengineering, and the Arizona Technology and Research Initiative Fund Imaging Fellowship, during the conduct of the study. In addition, all authors have a patent Falloposcope System and Method for Ovarian Cancer Detection pending.

\section{Acknowledgments}

The authors thank Dr. Khanh Kieu for his expertise and assistance tapering the illumination fiber. This work was supported by the United States Department of Defense (Grant No. W81XWH-13-1-0131), the National Institute of Biomedical Imaging and Bioengineering (Grant No. U54EB015403), and the Arizona Technology and Research Initiative Fund Imaging Fellowship.

\section{References}

1. American Cancer Society, "Cancer facts \& figures 2016," American Cancer Society, Atlanta, 2016, http://www.cancer.org/acs/groups/content/ @ research/documents/document/acspc-047079.pdf.

2. I. J. Jacobs et al., "Ovarian cancer screening and mortality in the UK collaborative trial of ovarian cancer screening (UKCTOCS): a randomised controlled trial," Lancet 387(10022), 945-956 (2015).

3. S. S. Buys et al., "Ovarian cancer screening in the prostate, lung, colorectal and ovarian (PLCO) cancer screening trial: findings from the initial screen of a randomized trial," Am. J. Obstet. Gynecol. 193(5), 1630-1639 (2005).

4. S. Vaughan et al., "Rethinking ovarian cancer: recommendations for improving outcomes," Nat. Rev. Cancer 11(10), 719-725 (2011).

5. P. O. Brown and C. Palmer, "The preclinical natural history of serous ovarian cancer: defining the target for early detection," PLoS Med. 6(7), e1000114 (2009).

6. E. Lengyel, "Ovarian cancer development and metastasis," Am. J. Pathol. 177(3), 1053-1064 (2010).

7. K. Eagle and J. A. Ledermann, "Tumor markers in ovarian malignancies," Oncologist 2(5), 324-329 (1997).

8. B. C. Vanderhyden, "Loss of ovarian function and the risk of ovarian cancer," Cell Tissue Res. 322(1), 117-124 (2005).

9. A. M. Karst, K. Levanon, and R. Drapkin, "Modeling high-grade serous ovarian carcinogenesis from the fallopian tube," Proc. Natl. Acad. Sci. U. S. A. 108(18), 7547-7552 (2011).

10. E. Kuhn, R. J. Kurman, and I.-M. Shih, "Ovarian cancer is an imported disease: fact or fiction?" Curr. Obstet. Gynecol. Rep. 1(1), 1-9 (2012).

11. E. Lorenzen et al., "A review of the human vs. porcine female genital tract and associated immune system in the perspective of using minipigs as a model of human genital Chlamydia infection," Vet. Res. 46, 116 (2015).

12. J. Kerin et al., "Development and application of a falloposcope for transvaginal endoscopy of the fallopian tube," J. Laparoendoscopic Surg. 1(1), 47-56 (1990).

13. A. Y. Wong and S. M. Walker, "Falloposcopy - a prerequisite to the proper assessment of tubal infertility," Hong Kong Med. J. 5(1), 76-81 (1999).

14. J. Kerin et al., "Falloposcopy: a microendoscopic technique for visual exploration of the human fallopian tube from the uterotubal ostium to the fimbria using a transvaginal approach," Fertil. Steril. 54(3), 390-400 (1990).

15. Y. Tanaka et al., "Renaissance of surgical recanalization for proximal fallopian tubal occlusion: falloposcopic tuboplasty as a promising therapeutic option in tubal infertility," J. Minim. Invasive Gynecol. 18(5), 651-659 (2011).

16. M. Waeny and C. S. Awaiba, "Mini camera modules pave way for less-invasive visualization," http://www.photonics.com/Article.aspx? AID=56663 (21 September 2016).

17. C. M. Lee et al., "Scanning fiber endoscopy with highly flexible, 1-mm catheterscopes for wide-field, full-color imaging," J. Biophotonics 3(5-6), 385-407 (2010).

18. E. J. Seibel et al., "In vivo laser-based imaging of the human fallopian tube for future cancer detection," Proc. SPIE 9304, 93040Q (2015).

19. R. George et al., "Parallel factor analysis of ovarian autofluorescence as a cancer diagnostic," Lasers Surg. Med. 44(4), 282-295 (2012).

20. R. A. Bhat et al., "Autofluorescence of normal, benign, and malignant ovarian tissues: a pilot study," Photomed. Laser Surg. 27(2), 325-335 (2009).

21. M. Brewer et al., "Fluorescence spectroscopy for in vivo characterization of ovarian tissue," Lasers Surg. Med. 29(2), 128-135 (2001).

22. M. A. Brewer et al., "Imaging of the ovary," Technol. Cancer Res. Treat. 3(6), 617-627 (2004).

23. T. E. Renkoski, K. D. Hatch, and U. Utzinger, "Wide-field spectral imaging of human ovary autofluorescence and oncologic diagnosis via previously collected probe data," J. Biomed. Opt. 17(3), 036003 (2012).

24. J. N. McAlpine et al., "Autofluorescence imaging can identify preinvasive or clinically occult lesions in fallopian tube epithelium: a promising step towards screening and early detection," Gynecol. Oncol. 120(3), 385-392 (2011).

25. T. H. Tate et al., "Multispectral fluorescence imaging of human ovarian and fallopian tube tissue for early-stage cancer detection," J. Biomed. Opt. 21(5), 056005 (2016).

26. C. Yang et al., "Target-to-background enhancement in multispectral endoscopy with background autofluorescence mitigation for quantitative molecular imaging," J. Biomed. Opt. 19(7), 076014 (2014). 
27. C. Yang et al., "Scanning fiber endoscope with multiple fluorescencereflectance imaging channels for guiding biopsy," Proc. SPIE 8936, 89360R (2014).

28. D. Lorenser et al., "Ultrathin side-viewing needle probe for optical coherence tomography," Opt. Lett. 36(19), 3894 (2011).

29. D. Lorenser et al., "Dual-modality needle probe for combined fluorescence imaging and three-dimensional optical coherence tomography," Opt. Lett. 38(3), 266-268 (2013).

30. R. A. Wall and J. K. Barton, "Fluorescence-based surface magnifying chromoendoscopy and optical coherence tomography endoscope," J. Biomed. Opt. 17(8), 086003 (2012).

31. L. P. Hariri et al., "Simultaneous optical coherence tomography and laser induced fluorescence imaging in rat model of ovarian carcinogenesis," Cancer Biol. Ther. 10(5), 438-447 (2010).

32. A. R. Tumlinson et al., "Miniature endoscope for simultaneous optical coherence tomography and laser-induced fluorescence measurement," Appl. Opt. 43(1), 113-121 (2004).

33. Y. T. Pan et al., "Enhancing early bladder cancer detection with fluorescence-guided endoscopic optical coherence tomography," Opt. Lett. 28(24), 2485-2487 (2003).

34. M. Keenan et al., "Design and characterization of a combined OCT and wide field imaging falloposcope for ovarian cancer detection," Biomed. Opt. Express 8(1), 124-136 (2017).

35. J. F. Black et al., "A six-color four-laser mobile platform for multispectral fluorescence imaging endoscopy," Proc. SPIE 9304, 93040T (2015).

36. E. Swan et al., "Stray light mitigation in a novel endoscope for fallopian tubes," Proc. SPIE 9304, 930410 (2015).

37. J.-H. Han, J. Lee, and J. U. Kang, "Pixelation effect removal from fiber bundle probe based optical coherence tomography imaging," Opt. Express 18(7), 7427-7439 (2010).

38. L. Gao and R. T. Smith, "Optical hyperspectral imaging in microscopy and spectroscopy-a review of data acquisition," J. Biophotonics 8(6), 441-456 (2015).

39. "Guidelines on limits of exposure to ultraviolet radiation of...: health physics," LWW, http://journals.lww.com/health-physics/ Fulltext/2004/08000/GUIDELINES_ON_LIMITS_OF_EXPOSURE_ TO_ULTRAVIOLET.6.aspx (8 February 2017).
Tyler $\mathbf{H}$. Tate received his BA degree in mathematics and philosophy from the University of Portland and his PhD from the College of Optical Sciences at the University of Arizona. His research focuses on multispectral fluorescence and optical coherence imaging of ovarian cancer. This includes the optical design and fabrication of dual modality miniature endoscopes with the ultimate goal of providing a minimally invasive screening method for early-stage ovarian cancer detection.

Molly Keenan received her BS degree in biomedical engineering from Boston University and her PhD in biomedical engineering from the University of Arizona. Her research focuses on the design, development, and preclinical validation of devices using optical techniques (optical coherence tomography, fluorescence imaging, and spectroscopy) for the early detection of cancer.

John Black is the founder of Glannaventa, Inc., a start-up company developing an imaging microendoscope for point-of-care early-stage ovarian cancer diagnosis. His interests include laser design and development, fiber optics, transdermal physiological monitoring, imaging catheters, optical diagnostics, and medical devices. He is a senior member of the IEEE and chair of the IEEE in Medicine and Biology Society-Santa Clara Valley Section.

Urs Utzinger is currently an associate professor and associate department head in biomedical engineering at the University of Arizona. He holds appointments in Ob/Gyn, optical sciences, electrical and computer engineering, and the $\mathrm{BIO5}$ Institute. His work on fiber-optic probes, diagnostic spectroscopy, and microcopy has led to more than 70 publications in peer-reviewed journals.

Jennifer K. Barton is a professor of biomedical engineering and interim director of the $\mathrm{BIO} 5$ Institute at the University of Arizona. Her research focuses on the development of miniature multimodality optical endoscopes for early detection of cancer, including instrumentation design, preclinical experiments, and translation to human pilot clinical studies. 